\title{
Computational modelling of gas-liquid multiphase flows with DQMOM and the N-phase Algebraic Slip Model
}

\author{
K. Swiderski ${ }^{1,2}$, D. Caviezel ${ }^{1}$, M. Labois ${ }^{1}$, D. Lakehal $^{1} \&$ \\ C. Narayanan ${ }^{1}$ \\ ${ }^{1}$ ASCOMP GmbH, Switzerland \\ ${ }^{2}$ Institute of Fluid Dynamics, Switzerland
}

\begin{abstract}
In the present work the Direct Quadrature Method of Moments (DQMOM) has been implemented into the CFD code TransAT. The TransAT code is a finite volume solver, based on structured multiblock grids, with a focus on multiphase flow modelling: including two-phase interface tracking, Lagrangian particle tracking and multiphase mixtures with an algebraic slip model generalized for an arbitrary number of phases (N-phase ASM). The DQMOM technique was combined with the turbulent N-phase ASM model in order to extend its ability to handle dispersed phase populations with each class having its own velocity field. In the scope of this work the DQMOM implementation has been validated by performing $0 \mathrm{D}, 1 \mathrm{D}$ and $2 \mathrm{D}$ test cases - from unit tests to very complex problems such as bubble columns. The drag force acting on the bubble population, turbulent dispersion, bubble aggregation, breakage and growth phenomena were considered. The results show that DQMOM is an efficient method for solving complex multiphase problems, and allows more sophisticated modelling than ASM. However attention should be paid to proper implementation into a CFD framework, especially when it comes to mass conservation, realizability of DQMOM abscissas and discretization of stiff particle interaction kernels.

Keywords: DQMOM, multiphase flow, mixture model, population balance, bubble column.
\end{abstract}




\section{Introduction}

In a typical bubbly flow application, various coupled phenomena can occur simultaneously, such as bubble coalescence and breakup due to interactions between bubbles. Almost all interactions occur in the presence of turbulence or hydrodynamic mixing, which usually dictate their rate. Under such conditions, the assumption of constant bubble size may lead to incorrect predictions of gas-liquid multiphase flow behaviour. The evolution and creation of bubbles of different sizes requires the representation of the particle size distribution function. This is typically achieved by the Population Balance Models (PBM), where the statistical distribution of the dispersed phase can be tracked. The $\mathrm{N}$-phase model with algebraic slip between phases as implemented in TransAT [1] needs to be extended in this regard. Since the N-phase model solves for the volume fraction of each phase, a natural way to extend it would be direct discretization of the distribution into a number of size bins with birth and death terms accounting for processes such as breakup, coalescence, growth, etc. [2, 3].

Among the other available PBM methods, such as the Method of Moments (MOM), Quadrature Method of Moments (QMOM) and their different variants [4], Direct Quadrature Method of Moments (DQMOM) [5] is chosen in this study. The main reason to not use the direct discretization method is the fact that moment-based methods are more efficient in capturing the distribution function [6]. It has been shown that whereas 12-18 bins were required for the direct discretization method, equal or more accurate results were obtained with only 6 moments using the QMOM method. However, the QMOM method remains difficult to implement because the moment equations are directly solved, whose closure requires the recreation of the quadrature nodes and weights from the moments. Also, the velocity field associated with a given moment is difficult to interpret if it depends strongly on the internal coordinate of the population balance equation; as is typically the case for bubbles of widely different sizes.

DQMOM, on the other hand, directly solves for the nodes and weights of the quadrature approximation instead of the chosen moments, and is therefore easier to implement, still offering good accuracy at acceptable computational effort [7]. This is important in the N-phase context wherein the population balance might need to be solved for multiple dispersed phases. This paper presents the implementation of DQMOM into TransAT along with several validation cases and application to a bubble column.

\section{Mathematical model}

In order to describe the dynamics of N-phase flows an Algebraic Slip Model (ASM) extended to N-phases is used in the present work. Within this framework the DQMOM method is used to capture the most important properties of the dispersed phase like bubble size, void fraction or interfacial area. In this study, only the population of one of the phases is represented using DQMOM. 


\subsection{N-phase Algebraic Slip Model}

The N-phase ASM represents multiphase flow in an ensemble averaged sense, where the involved phases move at different or equal velocities under the assumption of equilibrium within short spatial length scales [1]. The model is given in the form of one mass conservation equation for each of the $\mathrm{N}$ phases and one momentum conservation equation for the mixture, see eqns (1), (2) below. Momentum conservation equation may contain additional terms to account for velocity differences between the phases. The relative velocities must be computed from the force balance for the dispersed phases. The N-phase ASM for incompressible isothermal flow reads as follows:

$$
\begin{gathered}
\frac{\partial \rho_{k} \alpha_{k}}{\partial t}+\frac{\partial}{\partial x_{j}}\left[\rho_{k} \alpha_{k}\left(u_{j}^{(m)}+u_{j k}^{(d)}+\tilde{u}_{j}^{(t d)}\right)\right]=\frac{\partial}{\partial x_{j}}\left(\frac{v_{t}}{S c_{t}} \frac{\partial \rho_{k} \alpha_{k}}{\partial x_{j}}\right) \\
\frac{\partial}{\partial t}\left(\rho_{m} u_{i}^{(m)}\right)+\frac{\partial}{\partial x_{j}}\left(\rho_{m} u_{i}^{(m)} u_{j}^{(m)}\right)=-\frac{\partial p}{\partial x_{i}}+\frac{\partial}{\partial x_{j}}\left[2\left(\mu_{m}+\mu_{t}\right) S_{i j}^{(m)}\right] \\
-\frac{\partial}{\partial x_{j}}\left(\sum_{k=1}^{N} \alpha_{k} \rho_{k} u_{i k}^{(d)} u_{j k}^{(d)}\right)+\rho_{m} g_{i}+M^{\sigma} .
\end{gathered}
$$

The velocity associated with the phase $k$ is a sum of mixture, drift and turbulent drift contributions coming from components $u_{j k}=u_{j}^{(m)}+u_{j k}{ }^{(d)}+\tilde{u}_{j}^{(t d)}$ respectively. In the above equations, $\alpha_{k}$ is the volume fraction of phase $k$, $\rho_{m}=\sum_{k=1}^{N} \rho_{k} \alpha_{k}$ is the mixture density, $p=\sum_{k=1}^{N} \alpha_{k} p_{k}$ is the mixture pressure,

$S_{i j}{ }^{(m)}$ is the shear rate tensor for the mixture, $\mu_{m}$ is the mixture viscosity given by a rheological law, $\mu_{t}$ is the turbulent eddy viscosity, $g_{i}$ is gravitational acceleration, and $M^{\sigma}$ represents the effect of surface tension (ignored in our consideration). If $l$ is the dispersed phase and $k$ is the continuous phase, then the drift velocity of phase $k$ is given as $u_{j k}^{(d)}=\sum_{l=1}^{N} Y_{l} u_{j l k}{ }^{(s)}$, where $Y_{l}$ denotes mass fraction of phase $l$, and $u_{j l k}{ }^{(s)}$ is the slip between the phases $k$ and $l$ given by:

$$
-\frac{3}{8} \rho_{k} \frac{C_{d}}{R_{l}}\left|u_{j l k}{ }^{(s)}\right| u_{j l k}{ }^{(s)}=\left(\rho_{m}-\rho_{l}\right) g_{j},
$$

which represents the balance between the drag and buoyancy forces. Here $C_{d}$ is the drag coefficient and $R_{l}$ is the radius of the dispersed phase. Note that for the ASM model, $R_{l}$ is an input for each phase and remains constant.

For incompressible flows, the mixture continuity equation is given as,

$$
\frac{\partial u_{j}^{(m)}}{\partial x_{j}}+\frac{\partial \tilde{u}_{j}^{(t d)}}{\partial x_{j}}+\frac{\partial}{\partial x_{j}}\left(\sum_{k=1}^{N} \alpha_{k} u_{j k}^{(d)}\right)=0,
$$


where $\tilde{u}_{j}^{\left({ }^{(t)}\right)}=v_{t} \nabla_{j} \rho_{m} /\left(S c_{t} \rho_{m}\right)$ is the turbulent drift correction, and $v_{t} / S c_{t}$ denotes the mixture turbulent diffusivity, $S c_{t}$ is the turbulent Schmidt number, and $\mu_{t}=v_{t} \rho_{m}$ is mixture eddy viscosity. Eqn (4) represents the global volume balance and is used to derive the pressure correction equation. Turbulence is modelled with the standard $k-\varepsilon$ model for the mixture.

\subsection{Direct Quadrature Method of Moments}

DQMOM has recently become a very attractive approach for solving population balance equation (PBE) thanks to its capability of representing the most interesting properties of the population, e.g. mean diameter, void fraction, and number of particles. For many physical processes the representation of different phases simply by a volume fraction is not sufficient - especially problems involving phase change or mass transfer between involved phases, where the dispersed particles size changes.

The main idea behind using DQMOM is based on direct tracking of the weights $\omega_{c}$ and weighted abscissas $\omega_{c} \xi_{c}$ appearing in the quadrature approximation for the first $2 N_{c}$ moments of the discrete phase distribution. In the univariate version, DQMOM is developed for $N_{c}$ classes of internal variable (abscissa) $\xi_{c}$, which in our case represents the dispersed phase diameter. The volume fraction of the $c$-th class is given as $\alpha_{c}=k_{V} \omega_{c} \xi_{c}{ }^{3}$ and the shape factor $k_{V}=\pi / 6$ for spherical particles.

The following system:

$$
\begin{aligned}
& \frac{\partial \omega_{c}}{\partial t}+\frac{\partial}{\partial x_{j}} u_{j c} \omega_{c}=\frac{\partial}{\partial x_{j}}\left(D_{x} \frac{\partial \omega_{c}}{\partial x_{j}}\right)+a_{c}, \\
& \frac{\partial}{\partial t} \omega_{c} \xi_{c}+\frac{\partial}{\partial x_{j}} u_{j c} \omega_{c} \xi_{c}=\frac{\partial}{\partial x_{j}}\left(D_{x} \frac{\partial}{\partial x_{j}} \omega_{c} \xi_{c}\right)+b_{c}, \quad c=1, \ldots, N_{c},
\end{aligned}
$$

is solved with the use of the implicit solver in TransAT. When there is no inhomogeneity of the spatial distribution of abscissas (particle size $\xi_{c}$ for $c$-th class is not changing in space $\mathbf{x}$ ), the right hand side of eqn (5) can be simply set to zero. Otherwise the linear system (6) is solved with respect to diffusion correction term only $C_{k}$. Let us recall that the velocity associated with $c$-th class is given in the same way as in eqn (2) for $k$-th phase as, $u_{j c}=u_{j}^{(m)}+u_{j c}{ }^{(d)}+\tilde{u}_{j}^{(t d)}$.

The algebraic linear system for the RHS terms of eqn (5), namely $a_{c}$ and $b_{c}$, emerging from DQMOM framework reads as follows:

$$
(1-k) \sum_{c=1}^{N_{c}} \xi_{c}^{k} a_{c}+k \sum_{c=1}^{N_{c}} \xi_{c}^{k-1} b_{c}=S_{k}+C_{k}, \quad k=0, \ldots, 2 N_{c}-1 .
$$

Here $C_{k}=k(k-1) D_{x} \sum_{c=1}^{N_{c}}\left(\xi_{c}{ }^{k-1} \omega_{c} \partial_{x} \xi_{c} \cdot \partial_{x} \xi_{c}\right)$ is the diffusion correction source term for $k$-th moment of the distribution. $D_{x}$ is the diffusivity in physical space and represents any diffusion source that may affect the simulation, such as 
turbulent diffusion, molecular diffusion or numerical diffusion. The set of eqns (6) must be solved in each computational cell.

During the implementation of DQMOM into any CFD code, one can meet some difficulties regarding the aforementioned algebraic linear system illconditioning. This especially can happen when two or more abscissas are not sufficiently distinct from each other within the same computational cell (singularity problem), and/or the weights or abscissas values tend to zero values. To overcome this problem we use a condition number based algorithm to detect singularities, and if they appear, we use local averaging from neighbouring cells for the source term calculation. For example if reciprocal of the condition number is lower than $10^{-12}$, we consider the matrix as ill-conditioned. The method to deal with very low values of the weights can be enforcing a certain fractional number of particles per unit volume, that the weights directly represent themselves, e.g. at least 1-5 particles so that their impact on the mixture composition is negligible for practical applications.

In order to couple the stiff kernels with the convection-diffusion step of the equations, we employ the fractional time stepping technique. In the first step we solve the system of eqn (5) without considering physical processes source term $S_{k}$. For the details how to compute kernels term $S_{k}$ the reader can refer to [5]. In the second step we solve for physical processes like coalescence and breakage with the use of an ODE solver. Furthermore we use first-order scheme for each equation in the implicit solver to avoid problems with non-conservation as reported in [8].

As was already described, due to time scale of coalescence and breakage processes, the system created by the kernels is often stiff. In order to deal with it, we use the Variable-coefficient Ordinary Differential Equation solver, with fixed-leading-coefficient implementation (DVODE) [9]. The solver calculates the following set of equations:

$$
\frac{\partial \omega_{c}^{*}}{\partial t}=a_{c}^{*}, \quad \frac{\partial\left(\omega_{c}^{*} \xi_{c}^{*}\right)}{\partial t}=b_{c}^{*}, \quad c=1, \ldots, N_{c},
$$

where $a^{*}{ }_{c}$ and $b^{*}{ }_{c}$ are evaluated from eqn (6) by considering physical processes present in $S_{k}$ term. The $(*)$ denotes intermediate state of the solution obtained after the convection-diffusion step. Here the $C_{k}$ term is not considered since it is already applied in convection-diffusion update - diffusion time scale is typically much larger than breakage and coalescence processes.

In general, it can be shown that in the absence of dispersed phase interactions (DQMOM nodes remain spatially constant), the volume fraction advected via the ASM model and the volume fraction calculated for a given DQMOM class should match exactly.

\section{Validation and verification}

The validation and verification of DQMOM implementation have been done in several steps similar to the basic solution algorithm where the advectiondiffusion effects and the effect of source terms are solved through operator 
splitting. First we validated homogeneous problems for DQMOM in zerodimensional space (unit test) where advection is not considered. Particles aggregation, breakage and growth have been considered and the obtained results were compared to the reference data [5]. We also tested mass transfer between dispersed and continuous phase in one test case, for the system of ozone and water. The next step is the verification of convection and diffusion of DQMOM weights and nodes, where the solution is compared to the ASM method; in the absence of bubble interactions, an exact match is expected.

\subsection{Unit tests for the kernels}

In order to validate DQMOM implementation several unit tests have been performed. Here we point out only basic cases, testing simultaneous aggregation and breakage; molecular growth and mass transfer for a spatially homogeneous system.

Firstly a homogeneous population of particles suspended in a fluid is modelled. At the initial state we set $\omega_{1}=1, \omega_{2}=\omega_{3}=0.01$ and $\xi_{1}=1, \xi_{2}=2, \xi_{3}=1.5$. The particles undergo hydrodynamic aggregation $\left(\beta_{c \gamma}=\xi_{c}{ }^{3}+\xi_{\gamma}{ }^{3}\right)$ and power-law breakage $\left(a_{c}=0.02 \xi_{c}{ }^{3}\right)$ with symmetric fragmentation $\left(\bar{b}_{c}{ }^{(k)}=2^{(3-k) / 3} \xi_{c}{ }^{k}\right)$. The evolution of the Sauter mean diameter is shown in fig. 1, where an exact match with reference data [5] is obtained.

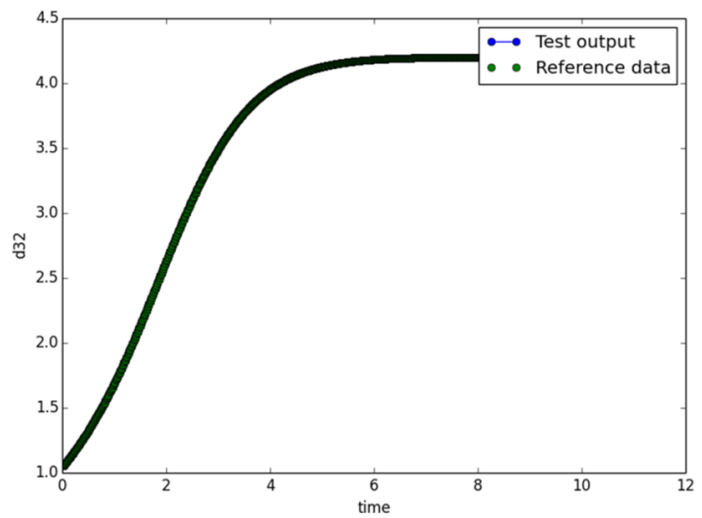

Figure 1: Hydrodynamic aggregation and power-law breakage with symmetric fragmentation with 3 classes.

Next we test DQMOM against molecular growth of mean crystal size with power-law kernel $\left(G(L)=L^{r}\right)$. Initial conditions are set according to moments set $m_{0}=1, m_{1}=1 / 2, m_{2}=1 / 3, m_{3}=1 / 4$. The result exactly matches reference data [5] as shown in fig. 2. 
In the further step we test the dissolution of ozone in water. It should be noted that not only does the bubble size reduce with time due to dissolution, but the mass transfer rate is also a function of the bubble size given by,

$$
\dot{m}=\frac{d C_{l}}{d t}=k_{l} a\left(C_{l}-C_{l}^{s a t}\right)
$$

where $k_{l}=1.13\left(D\left|V_{s}\right| / d_{g}\right)^{0.5}$ is the liquid phase mass transfer coefficient $(\mathrm{m} / \mathrm{s})$ calculated according to the Higbie model and $a=6 \alpha_{g} / d_{g}$ is interfacial area per unit volume $\left(\mathrm{m}^{2} / \mathrm{m}^{3}\right)$ assuming spherical bubbles. We assume a constant slip velocity $V_{s}$ equal $0.2 \mathrm{~m} / \mathrm{s}$, initial bubble size $d_{g}$ of $2 \mathrm{~mm}$, ozone molecular diffusivity $D=1.4 \mathrm{E}-9 \mathrm{~m}^{2} / \mathrm{s}$ and saturation concentration of ozone in water $C_{l}^{\text {sat }}=$ $0.004 \mathrm{~kg} / \mathrm{m}^{3}$. These values should be reasonable for ozone-water system under normal conditions. The evolution of the mass transfer rate with time is presented in fig. 3. Here one can observe noticeable discrepancies with respect to reference solution. This happens because the time step used for explicit integration of eqn (8), in order to obtain the reference solution, was set to arbitrarily high value of $1 \mathrm{~s}$.

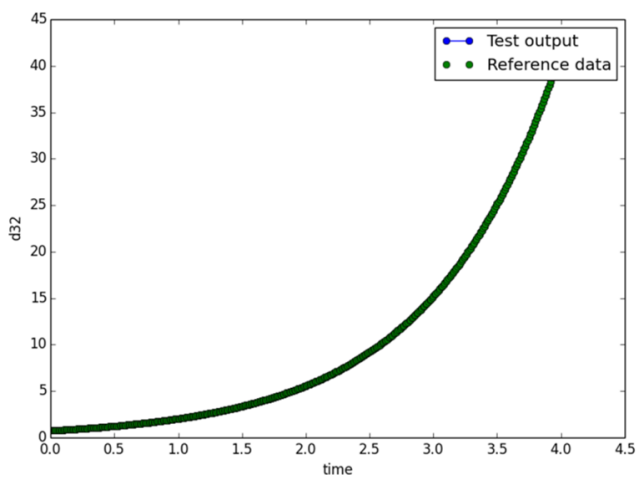

Figure 2: Homogeneous molecular growth. Power law growth with 2 classes, $\mathrm{r}=1$.

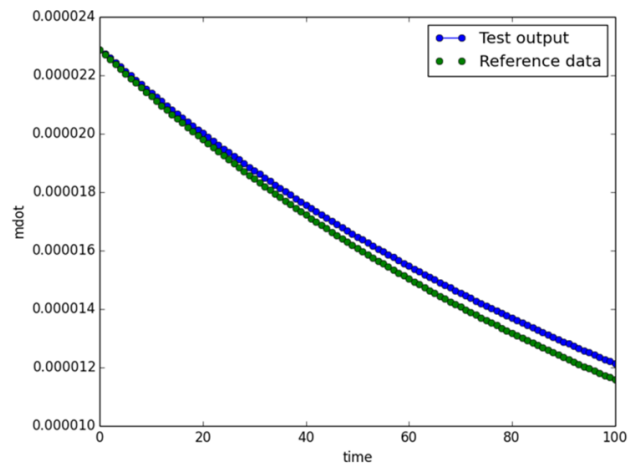

Figure 3: Homogeneous mass transfer. Dissolution with 2 classes. 


\subsection{Verification of convection: phase separation problem in 1D}

A separation tank is simulated with gravity-driven phase separation (see fig. 4) between water and air phases. Initially 1/10th part of the tank is filled from the left side with air and water assuming equal volume fractions. The rest part contains water only. We split the air into 2 classes for DQMOM and into 2 phases for ASM. We assume that the number of bubbles is the same for each class at initial state (weights are equal $\omega_{1}=\omega_{2}$ ). From this assumption the initial gas volume fractions are evaluated to be $\alpha_{2}=0.06$ and $\alpha_{3}=0.44$. The bubble sizes are initialized to $1 \mathrm{~mm}$ and $2 \mathrm{~mm}$ respectively. For DQMOM the initial bubble sizes should remain unchanged during the simulation, whereas in ASM the bubble size is not solved for. The slip between the phases is calculated considering the balance between drag force and buoyancy. Boundary conditions used in the simulation are slip walls. The simulation was run for $60 \mathrm{~s}$. The variation in gas volume fraction along the tank is shown in fig. 5. As expected, the gas phases segregate from each other due the difference in the slip velocities given by the different bubble sizes; the larger bubbles rise faster. The ASM and DQMOM simulations give exactly the same results.

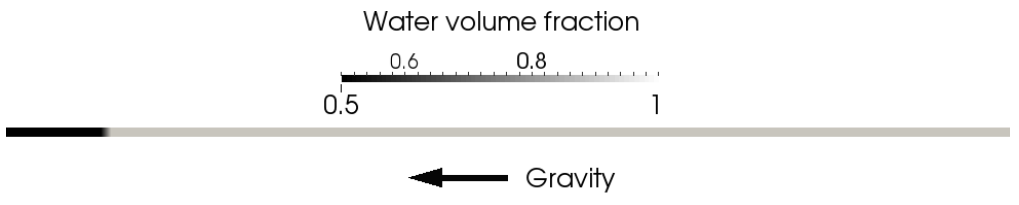

Figure 4: 1D test case with phase separation. Initial state.

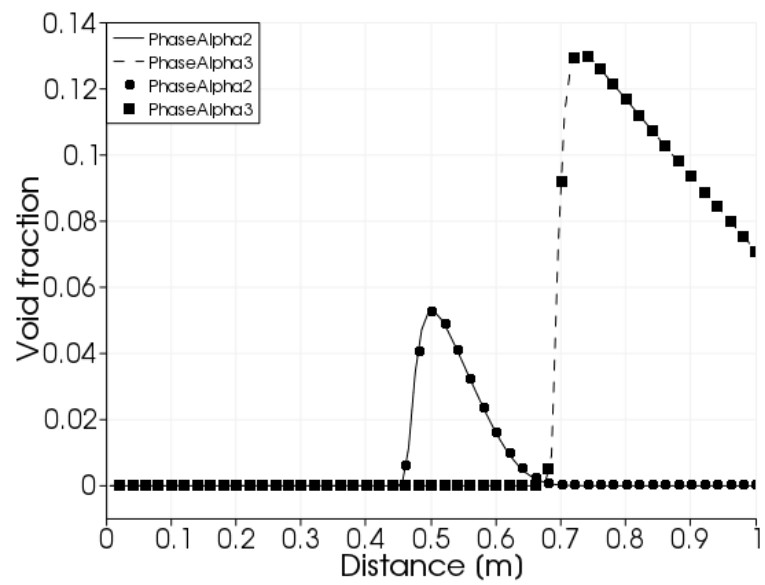

Figure 5: 1D phase separation problem: gas void fractions. Markers denote DQMOM solution, lines - ASM. 


\section{Bubble column}

In order to test the DQMOM implementation for a real problem including advection effects and interaction processes, a two-dimensional bubble column has been chosen. The configuration of the problem is similar as in Buffo et al. [10]. Air is injected into stagnant water from the bottom side of the tank with the velocity of $1.4236 \mathrm{~m} / \mathrm{s}$. The boundary conditions are no-slip walls everywhere and pressure outlet at the top. The tank has a height of $2.16 \mathrm{~m}$, width $0.63 \mathrm{~m}$ and air inflow width is set to $0.033 \mathrm{~m}$. A rectangular non-uniform grid with approximately 11,000 cells is used. Initially the tank is filled with water up to $1.26 \mathrm{~m}$.

For the first 9s of the simulation we use the ASM model in order to reach a pseudo-steady state. A bubble size of $3.7 \mathrm{~mm}$ was used for the ASM calculation. Then the ASM result at 9s (see fig. 6) is used as initial condition for the DQMOM simulation. Here we consider bubble coalescence and breakage by employing Laakkonen kernels [11] with their default constants. Furthermore, the standard k- $\varepsilon$ model is employed here with standard wall functions. The inlet bubble population is assumed to have log-normal distribution with standard deviation $15 \%$ of mean diameter value $(3.7 \mathrm{~mm})$. We use two classes representation of the bubbles population. Turbulence intensity at the inlet is $10 \%$ and the eddy viscosity ratio is set arbitrarily to 200 . We consider drag and buoyancy forces acting on the bubbles as well as the turbulent dispersion effect. It is worth mentioning that each bubble class has its own slip velocity whose value depends locally on its size.
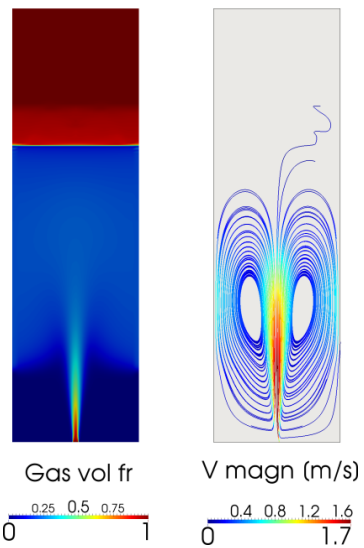

Figure 6: Instantaneous gas volume fraction and stream traces coloured by velocity magnitude at time 9s. Pseudo steady state obtained with the use of ASM, used as initial state for DQMOM simulation.

It is clearly seen in fig. 7 that bubble distribution size varies with preference for breakage processes near to the injection region. In the rest of the computational domain coalescence dominates and therefore mean bubble size 
increases. Because only gas is injected, the momentum of the jet is not strong enough to penetrate the whole water column and the jet velocity is close to zero in upper half of the tank. Due to this fact bubbles coalesce in low-velocity regions near the free surface and near the walls. The drift velocity and bubble size distribution field is shown in fig. 8. As expected the bubbles have different drift velocities which is the reason for bubble segregation in water. Fig. 9 shows the variation of the total gas volume fraction along the jet axis. Although the ASM results are very similar to the DQMOM results, the strong variation of bubble sizes indicates the utility of DQMOM in modelling exchange processes between the dispersed and continuous phases because, in general, they depend non-linearly on the bubble sizes.

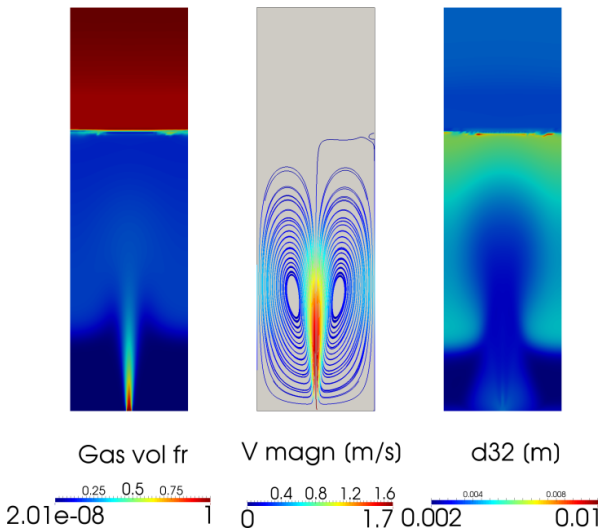

Figure 7: Instantaneous gas volume fraction, stream traces coloured by velocity magnitude and Sauter diameter at time 14s.

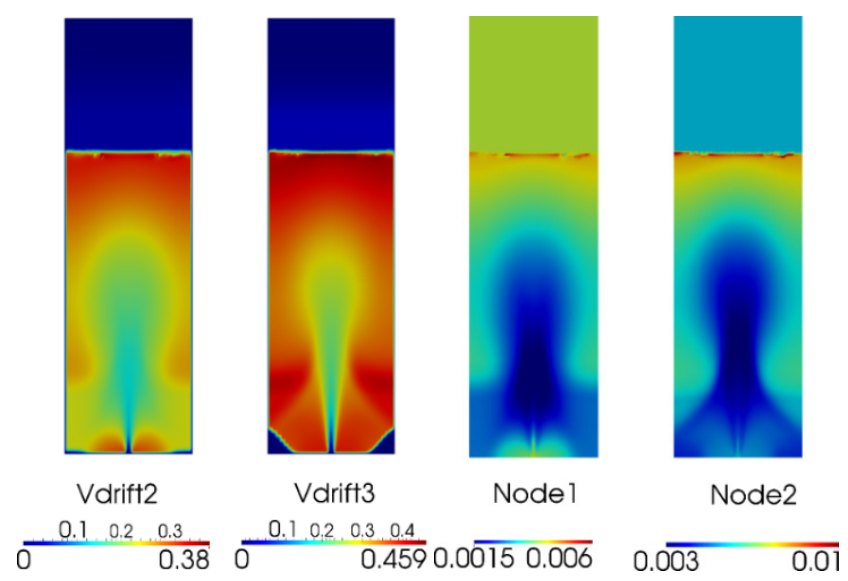

Figure 8: Instantaneous drift velocity $(\mathrm{m} / \mathrm{s})$ field contours and abscissas (nodes) (m) contours for two DQMOM classes at time 14s. 


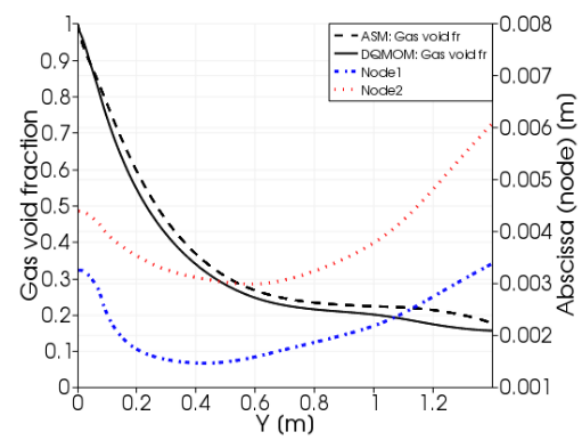

Figure 9: Instantaneous gas void fraction plot along the centreline Y (height of the tank) for ASM and DQMOM at time 9s and 14s respectively. Abscissas (nodes) (m) plots for two DQMOM classes at time 14s.

\section{Conclusions}

The paper describes fundamental details of N-phase ASM model and DQMOM. The first strategy is widely used for solving practical multiphase problems while the second one allows incorporating population balance in a feasible way and at a reasonable computational cost. Both methods are conservative for homogeneous and inhomogeneous problems. However special attention must be paid to proper implementation of DQMOM into a CFD framework. Especially for inhomogeneous systems, DQMOM formulation demands diffusion correction, as originally formulated in [5], to suppress non-conservation of moments higher than the first two. We have demonstrated that CFD-PBE based method is a powerful tool for solving complex problems like bubble columns. The next step will be to extend the model to a bubble column with mass transfer and validation by experimental data. From an application point of view, extension of the population balance model to handle multiple dispersed phases would be very useful.

\section{Acknowledgement}

This work is a part of the project TRIUMPH (Treating Urban Micropollutants and Pharmaceuticals in Wastewaters) realized in the framework of EUREKA intergovernmental network.

\section{References}

[1] Manninen, M., Taivassalo, V. \& Kallio, S., On the mixture model for multiphase flow. VTT Publications, 288, 1996.

[2] Yeoh, G.H., Cheung, S.C.P. \& Tu, J.Y., On the prediction of the phase distribution of bubbly flow in a horizontal pipe. Chemical Engineering Research \& Design, 90(1), pp. 40-51, 2012. 
[3] Frank, T., Zwart, P.J., Shi, J.-M., Krepper, E., Lucas, D. \& Rohde, U., Inhomogeneous MUSIG model - a population balance approach for polydispersed bubbly flows. Int. Conf. of Nuclear Energy for New Europe, Bled, Slovenia, 2005.

[4] Marchisio, D. \& Fox, R.O., Computational Models for Polydisperse Particulate and Multiphase Systems, Cambridge University Press, 2013.

[5] Marchisio, D. \& Fox, R.O., Solution of population balance equations using the direct quadrature method of moments. J. Aerosol Science, 36(1), pp. 43-73, 2005.

[6] Sanyal, J., Marchisio, D.L., Fox, R.O. \& Dhanasekharan, K., On the comparison between population balance models for CFD simulation of bubble columns. Ind. Eng. Chem. Res., 44(14), pp. 5063-5072, 2005.

[7] Cheung, S.C.P., Deju, L., Yeoh, G.H. \& Tu, J.Y., Modelling of bubble size distribution in isothermal gas-liquid flows: Numerical assessment of population balance approaches. Nuclear Engineering and Design, 265, pp. 120-136, 2013.

[8] Mazzei, L., Segregation dynamics of dense polydisperse fluidized suspensions modeled using a novel formulation of the direct quadrature method of moments. Chemical Engineering Science, 101, pp. 565-576, 2013.

[9] Brown, P.N., Byrne, G.D. \& Hindmarsh, A.C., VODE: A Variable Coefficient ODE Solver, SIAM J. Sci. Stat. Comput., 10, pp. 1038-1051, 1989. Also, LLNL Report UCRL-98412, 1988.

[10] Buffo, A., Vanni, M., Marchisio, D.L. \& Fox, R.O., Multivariate Quadrature-Based Moment Methods for turbulent polydisperse gas-liquid systems. Int. J. Multiphase Flow, 50, pp. 41-57, 2013.

[11] Laakkonen, M., Moilanen, P., Alopaeus, V. \& Aittamaa, J., Modelling local bubble size distributions in agitated vessels. Chemical Engineering Science, 62(3), pp. 721-740, 2007. 